\title{
Decomposition of the polynomial kernel of arbitrary higher spin Dirac operators
}

D. Eelbode, ${ }^{1, \text { a) }}$ T. Raeymaekers, ${ }^{2, \text { b) }}$ and J. Van der Jeugt ${ }^{3, c)}$

1) Department of Mathematics and Computer Science, University of Antwerp, Campus Middelheim, G-Building, Middelheimlaan 1, 2020 Antwerpen, Belgium

${ }^{2)}$ Clifford Research Group, Department of Mathematical Analysis, Ghent University, Galglaan 2, 9000 Ghent, Belgium

3) Department of Applied Mathematics, Computer Science and Statistics, Ghent University, Krijgslaan 281, 9000 Ghent, Belgium

(Dated: July 16, 2015)

In a series of recent papers, we have introduced higher spin Dirac operators, which are generalisations of the classical Dirac operator. Whereas the latter acts on spinorvalued functions, the former act on functions taking values in arbitrary irreducible half-integer highest weight representations for the spin group. In this paper, we describe how the polynomial kernel spaces of such operators decompose in irreducible representations of the spin group. We will hereby make use of results from representation theory.

PACS numbers: 02.20.Qs, 02.20.Sv, 02.30.Fn, 21.10.Hw

Keywords: Dirac operator, higher spin, kernel space, representations

\footnotetext{
a)Electronic mail: David.Eelbode@ua.ac.be

b)Electronic mail: Tim.Raeymaekers@UGent.be

c)Electronic mail: Joris.VanderJeugt@UGent.be
} 


\section{INTRODUCTION}

This paper deals with higher spin Dirac operators (or HSD operators for short). These are elliptic first order differential operators, generalising the classical (massless) Dirac operator known from particle physics ${ }^{9}$. HSD operators act on functions $f(x)$, defined on $\mathbb{R}^{m}$, which take values in arbitrary irreducible finite-dimensional half-integer highest weight representations for the spin group, or equivalently, for its Lie algebra $\mathfrak{s o}(m)$. We study these operators in the setting of Clifford analysis, as this framework allows us to construct such representations as well-described function spaces, making it possible to make use of explicit computations in several vector variables. This is a function theory which, on the one hand generalises classical complex analysis, while on the other hand, it is a refinement of harmonic analysis on $\mathbb{R}^{m 2,8,12}$.

The first generalisations of the Dirac operator are the Rarita-Schwinger operators ${ }^{3,4}$, another class of operators originating in particle physics ${ }^{16}$. These operators were further generalised in the more recent papers ${ }^{6,7,10}$. As for any function theory, the understanding of the kernel space for these operators is crucial, in particular the polynomial kernel spaces, as it is dense in the space of continuous functions. The aim of the present paper is to study the vector space of polynomial solutions for arbitrary higher spin Dirac operators as a spin group module. A first attempt was made in ${ }^{11}$, where the problem at hand was converted to a combinatorial problem. The main drawback of the approach in the article was that the procedure only worked if the degree of homogeneity of the polynomial solutions was large enough and the fact that the combinatorial problem at hand remained an open problem. In this paper, we describe a different approach, which works for all cases and fully solves the proposed problem. The main problem lies in the fact that HSD operators stand in sharp contrast to the classical Dirac operator. Whereas the space of polynomial solutions for the latter is multiplicity free, the solution spaces for the HSD operators are highly reducible and not multiplicity free.

The structure of this paper is as follows. We will introduce basic definitions and concepts of Clifford analysis in Section 2, which will allow us to define higher spin Dirac operators. In Section 3, we will introduce some results from the representation theory of classical Lie groups, more specifically about the decompositions of tensor products. Finally, in Section 4, we use these results determine the full decomposition of the polynomial kernels of higher 
spin Dirac operators.

\section{NOTATIONS AND DEFINITIONS}

The real universal Clifford algebra $\mathbb{R}_{m}$ is defined as an algebra over the $m$-dimensional real space $\mathbb{R}^{m}$ with standard orthonormal basis $\left\{e_{1}, \ldots, e_{m}\right\}$, governed by the multiplication relations $e_{i} e_{j}+e_{j} e_{i}=-2 \delta_{i j}$. The complex Clifford algebra $\mathbb{C}_{m}$ is the complexification of the real Clifford algebra $\mathbb{C}_{m}=\mathbb{R}_{m} \otimes \mathbb{C}$. Within $\mathbb{R}_{m}$, the spin group can be defined as the set of even products of unit vectors:

$$
\operatorname{Spin}(m):=\left\{\prod_{j=1}^{2 p} \omega_{j}: \omega_{j} \in S^{m-1}\right\},
$$

where $S^{m-1}$ is the unit sphere in $\mathbb{R}^{m}$. The Lie algebra of the spin group is the (real) orthogonal algebra $\mathfrak{s o}(m)$. One can realise the space of Dirac spinors $\mathbb{S}$ as a minimal left ideal in $\mathbb{C}_{m}$, but more importantly, $\mathbb{S}$ defines a model for a representation of the spin group. In case of an odd dimension $m=2 n+1$, it is the irreducible basic half-integer representation with highest weight $\left(\frac{1}{2}, \ldots, \frac{1}{2}\right)$, which has $n$ entries. In case of an even dimension $m=2 n$, the spinor space is reducible as spin-representation, $\mathbb{S}=\mathbb{S}^{+} \oplus \mathbb{S}^{-}$where the summands are irreducible representations for $\operatorname{Spin}(m)$ with highest weights $\left(\frac{1}{2}, \ldots, \frac{1}{2}\right)$ and $\left(\frac{1}{2}, \ldots, \frac{1}{2},-\frac{1}{2}\right)$. For this reason, we will restrict ourselves to odd dimensions throughout this article.

In general all irreducible representations of the spin group can be characterised by their highest weight, containing coordinates $\lambda=\left(l_{1}, \ldots, l_{n}\right)$ with respect to the standard basis $\left\{L_{j}, 1 \leq j \leq n\right\}$ for the dual space $\mathfrak{h}^{*}$. Here, $\mathfrak{h}$ denotes a Cartan algebra for the Lie algebra $\mathfrak{s o}(m)$.

At the heart of classical Clifford analysis lies the (massless) Dirac operator. This is a conformally invariant elliptic first order differential operator mapping functions taking values in the spinor space $\mathbb{S}$ to the same space. This operator is given by $\partial_{x}=\sum_{j=1}^{m} e_{j} \partial_{x_{j}}$. It factorises the $m$-dimensional Laplace operator $\Delta_{x}=-\partial_{x}^{2}$ which is the reason why Clifford analysis is often seen as a refinement of harmonic analysis. This implies that the space of polynomial solutions for the Dirac operator is a subspace of the space of $\mathbb{S}$-valued harmonic polynomials. The space of polynomials of degree $h$ in a vector variable $x$ will be denoted by $\mathcal{P}_{h}\left(\mathbb{R}^{m}, \mathbb{C}\right)$. When using variables $u_{j} \in \mathbb{R}^{m}$, the corresponding Euler operator, Laplace operator and Dirac operator will be denoted by $\mathbb{E}_{j}, \Delta_{j}$ and $\partial_{j}$ rather than $\mathbb{E}_{u_{j}}, \Delta_{u_{j}}$ and $\partial_{u_{j}}$. 
Decomposition of the polynomial kernel of arbitrary higher spin Dirac operators

The notation $\langle\cdot, \cdot\rangle$ is reserved for the Euclidean inner product on $\mathbb{R}^{m}$. As Dirac operators are very similar to vectors, we extend the Euclidean inner product, e.g. $\left\langle x, \partial_{y}\right\rangle=\sum_{j=1}^{m} x_{j} \partial_{y_{j}}$ for any two vectors $x=\left(x_{1}, \ldots, x_{m}\right)$ and $y=\left(y_{1}, \ldots, y_{m}\right)$

Within Clifford analysis, all finite dimensional irreducible representations of the spin group can be modeled by polynomial spaces ${ }^{5,12}$. Therefore, let us introduce the following definitions.

Definition II.1. A function $f: \mathbb{R}^{k m} \rightarrow \mathbb{C}:\left(u_{1}, \ldots, u_{k}\right) \mapsto f\left(u_{1}, \ldots, u_{k}\right)$ is called simplicial harmonic if it satisfies the system

$$
\begin{aligned}
& \left\langle\partial_{i}, \partial_{j}\right\rangle f=0, \text { for all } i, j=1, \ldots, k \\
& \left\langle u_{i}, \partial_{j}\right\rangle f=0, \text { for all } 1 \leq i<j \leq k
\end{aligned}
$$

The vector space $\mathcal{H}_{\lambda}=\mathcal{H}_{l_{1}, \ldots, l_{k}}$ is the space of $\mathbb{C}$-valued simplicial harmonic polynomials, homogeneous of degree $l_{i}$ in $u_{i}\left(\lambda=\left(l_{1}, \ldots, l_{k}\right)\right)$.

Definition II.2. A function $f: \mathbb{R}^{k m} \rightarrow \mathbb{S}:\left(u_{1}, \ldots, u_{k}\right) \mapsto f\left(u_{1}, \ldots, u_{k}\right)$ is called simplicial monogenic if it satisfies the system

$$
\begin{gathered}
\partial_{i} f=0, \text { for all } i=1, \ldots, k \\
\left\langle u_{i}, \partial_{j}\right\rangle f=0, \text { for all } 1 \leq i<j \leq k .
\end{gathered}
$$

The vector space $\mathcal{S}_{\lambda}=\mathcal{S}_{l_{1}, \ldots, l_{k}}$ is the space of $\mathbb{S}$-valued simplicial monogenic polynomials, homogeneous of degree $l_{i}$ in $u_{i}\left(\lambda=\left(l_{1}, \ldots, l_{k}\right)\right)$.

These polynomial spaces have the following properties (e.g. $\left.{ }^{5}\right)$.

Theorem II.3. Set $\lambda=\left(l_{1}, \ldots, l_{k}\right)$ where $l_{1} \geq l_{2} \geq \cdots \geq l_{k}$.

- The polynomial space $\mathcal{H}_{\lambda}$ is a model for the irreducible Spin(m)-representation with highest weight

$$
\left(l_{1}, \ldots, l_{k}, 0, \ldots, 0\right) \text {. }
$$

As the zeros at the end do not matter for the representation, we will simply denote this highest weight with $\lambda$.

- The polynomial space $\mathcal{S}_{\lambda}$ is a model for the irreducible Spin(m)-representation with highest weight

$$
\left(l_{1}+\frac{1}{2}, \ldots, l_{k}+\frac{1}{2}, \frac{1}{2}, \ldots, \frac{1}{2}\right)
$$

We will denote a highest weight of this type by $\lambda^{\prime}$. 
One can now consider smooth functions $f\left(x ; u_{1}, \ldots, u_{k}\right) \in \mathcal{C}^{\infty}\left(\mathbb{R}^{m}, \mathcal{S}_{\lambda}\right)$, taking values in the irreducible spin module $\mathcal{S}_{\lambda}$. This means that for any $x \in \mathbb{R}^{m}$ fixed, the resulting polynomial in the dummy variables $u_{j}$ satisfies the requirements stated in Definition II.2. On this type of functions, we can define conformally invariant differential operators which are direct generalisations of the Dirac operator: higher spin Dirac operators.

Definition II.4. For an arbitrary half-integer highest weight $\lambda^{\prime}$, the associated HSD operator $\mathcal{Q}_{\lambda}$ is given by

$$
\mathcal{Q}_{\lambda}:=\prod_{j=1}^{k}\left(1+\frac{u_{j} \partial_{j}}{m+2 \mathbb{E}_{j}-2 j}\right) \partial_{x}
$$

Note that the product is understood to be ordered, with indices increasing from left to right. The Euler operator in the variable $u_{j}$ acts as the constant $l_{j}$, as the operator acts on homogeneous polynomials. In the case where $\lambda=l_{1}$, this operator corresponds to the classical Rarita-Schwinger operator coming from physics ${ }^{16}$. When $\lambda=(0)$, this operator is the classical Dirac operator.

For the construction of this operator, we refer the reader to ${ }^{10}$.

As for any good function theory, we have to understand the polynomial kernel of such HSD operators. This means that we have to decompose the kernel into irreducible representations of the spin group. Contrary to the case of the classical Dirac operator however, we will see that this kernel is highly reducible and not multiplicity free.

\section{TENSOR PRODUCT DECOMPOSITIONS}

In order to continue our reasoning, we need to know a bit more about tensor product decompositions. We use the results found $\mathrm{in}^{1}$. In order to understand these results, we have to introduce some notations. Remember that any irreducible $\operatorname{Spin}(m)$-representation is in 1-1 correspondence with its highest weight. Therefore, we will from now on denote such representation by means of its highest weight. A general integer valued highest weight $\lambda=\left(l_{1}, \ldots, l_{k}\right)$ can be seen as a partition of $l=l_{1}+\cdots+l_{k}$, with the condition that $l_{1} \geq \cdots \geq l_{k}$. Each such partition $\lambda$ specifies a regular Young diagram $F^{\lambda}$ consisting of $l$ boxes arranged in $k$ left-adjusted rows. The length of the $j$-th row is $l_{j}$ for all $j=1, \ldots, k$. 
Decomposition of the polynomial kernel of arbitrary higher spin Dirac operators

For instance, the Young diagram for $(5,3,2,2)$ is given by

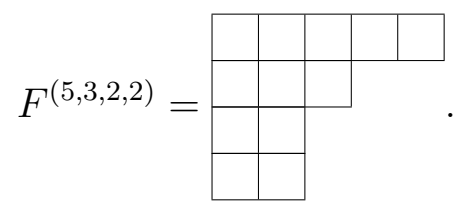

Take two partitions $\lambda=\left(\lambda_{1}, \ldots, \lambda_{a}\right)$ and $\mu=\left(\mu_{1}, \ldots, \mu_{b}\right)$. Then one defines a product on such partitions corresponding to the product of Schur functions $s_{\lambda} \cdot s_{\mu}{ }^{15}$, which is governed by the Littlewood-Richardson rule ${ }^{15}$ as follows:

$$
\lambda \cdot \mu=\sum_{\nu} m_{\lambda \mu}^{\nu} \nu
$$

where the coefficients $m_{\lambda \mu}^{\nu}$ are the number of distinctly labeled Young diagrams $F^{\nu}$ obtained from $F^{\lambda}$ by the addition of the boxes of $F^{\mu}$ in accordance with the following procedure: $\mu_{1}$ letters $a, \mu_{2}$ letters $b, \mu_{3}$ letters $c, \ldots$ are added alphabetically to $F^{\lambda}$, one letter at a time in such way that at every stage

(i) if the added letters are interpreted as boxes the resulting Young diagram is regular (each row at least as long as the next one),

(ii) no two identical letters appear in the same column,

(iii) the sequence of added letters read from right to left across each row in turn from top to bottom is a lattice permutation, in the sense that in this sequence the number of letters $a \geq$ then the number of letters $b \geq$ the number of letters $c \geq \cdots$ at every stage of the sequence.

Example III.1. Let us give an example of this rule, where the product of partitions $\lambda$ and $\mu$ is identified with the product of the corresponding Young diagrams $F^{\lambda}$ and $F^{\mu}$ :

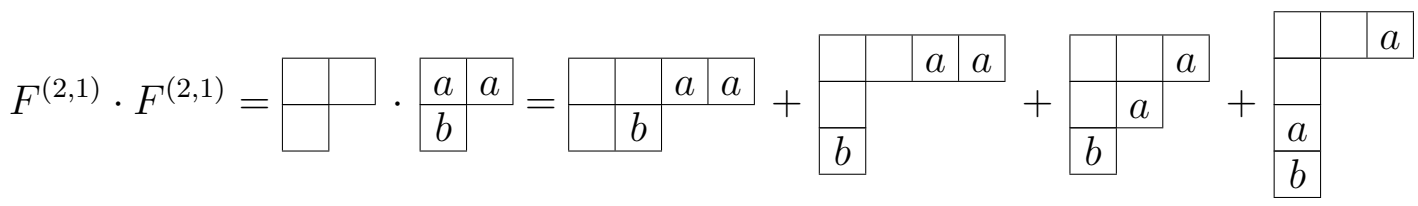

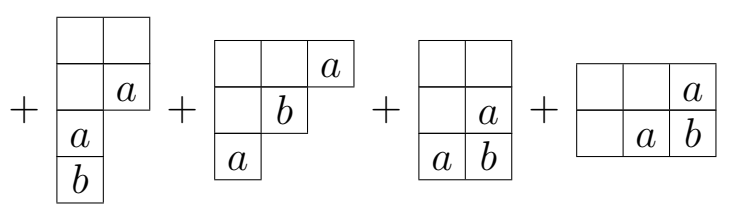

$$
\begin{aligned}
& =F^{(4,2)}+F^{(4,1,1)}+2 F^{(3,2,1)}+F^{(3,1,1,1)} \\
& +F^{(2,2,1,1)}+F^{(2,2,2)}+F^{(3,3)} \text {. }
\end{aligned}
$$


Another action we can perform on two partitions is the division, corresponding to the skew Schur functions ${ }^{15}$ :

$$
\nu / \mu=\sum_{\lambda} m_{\lambda \mu}^{\nu} \lambda
$$

The coefficients that appear are the Littlewood-Richardson coefficients we have introduced above. $\nu / \mu$ is the sum of those $\lambda$ whose product with $\mu$ yields $\nu$.

Let us now define $Q=\sum_{p=0}^{\infty}\left(1^{p}\right)$, the sum of all partitions where the Young diagram is a single column of length $p$. This is a so-called infinite S-function series ${ }^{1,13}$, and manipulations such as the product or the division of a partition by such a series are well defined.

With these definitions, we can formulate the following theorem.

Theorem III.2 $\left(^{1}\right)$. The tensor product of two $\operatorname{Spin}(m)$-representations with integer valued highest weights $\lambda$ and $\mu$ is given by

$$
\lambda \otimes \mu=\sum_{\zeta}(\lambda / \zeta) \cdot(\mu / \zeta)
$$

The tensor product of two $\operatorname{Spin}(m)$-representations where one has an integer valued highest weight $\lambda$ and the other has a half integer highest weight $\mu^{\prime}$ is given by

$$
\lambda \otimes \mu^{\prime}=\sum_{\zeta}((\lambda /(\zeta \cdot Q)) \cdot(\mu / \zeta))^{\prime}
$$

\section{KERNEL DECOMPOSITION}

The aim of this section is to arrive at an algorithm which tells us how to decompose the kernel of the higher spin Dirac operators into irreducible summands under the (regular) action of the spin group in an alternative way. We will make use of a higher spin version of the Cauchy-Kowalewskaya extension which was proven in ${ }^{11}$.

Theorem IV.1. As vector spaces, we have the isomorphism

$$
\mathcal{K}_{h, \lambda}:=\mathcal{P}_{h}\left(\mathbb{R}^{m}, \mathcal{S}_{\lambda}\right) \cap \operatorname{ker} \mathcal{Q}_{\lambda} \cong \mathcal{P}_{k}\left(\mathbb{R}^{m-1}, \mathcal{S}_{\lambda}\right)
$$

for any higher spin Dirac operator $\mathcal{Q}_{\lambda}$ corresponding to an arbitrary highest weight $\lambda$ of a half-integer irreducible representation of finite dimension. This means that each $h$ homogeneous polynomial in the kernel of $\mathcal{Q}_{\lambda}$ corresponds to an h-homogeneous $\mathcal{S}_{\lambda}$-valued polynomial in $m-1$ variables, and vice versa. 
Decomposition of the polynomial kernel of arbitrary higher spin Dirac operators

We can then make use of the classical Fischer decomposition for harmonic polynomials to obtain the direct sum formula

$$
\mathcal{P}_{h}\left(\mathbb{R}^{m-1}, \mathcal{S}_{\lambda}\right) \cong \mathcal{P}_{h}\left(\mathbb{R}^{m-1}, \mathbb{C}\right) \otimes \mathcal{S}_{\lambda} \cong\left(\bigoplus_{j=0}^{\left\lfloor\frac{h}{2}\right\rfloor} \mathcal{H}_{h-2 j}\left(\mathbb{R}^{m-1}, \mathbb{C}\right)\right) \otimes \mathcal{S}_{\lambda}
$$

On the other hand, we also have the classical branching rules for harmonic polynomials:

$$
\mathcal{H}_{h}\left(\mathbb{R}^{m}, \mathcal{S}_{\lambda}\right) \cong\left(\bigoplus_{j=0}^{h} \mathcal{H}_{j}\left(\mathbb{R}^{m-1}, \mathbb{C}\right)\right) \otimes \mathcal{S}_{\lambda}
$$

Combining both formulae, we arrive at the following result, which is a formal identity, to be understood on the level of isomorphisms. Note that we have omitted the space of values $\mathcal{S}_{\lambda}$, to shorten the notations:

$$
\begin{cases}h=2 \kappa & \mathcal{P}_{h}\left(\mathbb{R}^{m-1}\right) \cong \bigoplus_{j=0}^{\kappa} \mathcal{H}_{h-2 j}\left(\mathbb{R}^{m}\right) \backslash \bigoplus_{j=0}^{\kappa-1} \mathcal{H}_{h-(2 j+1)}\left(\mathbb{R}^{m}\right) \\ h=2 \kappa+1 & \mathcal{P}_{h}\left(\mathbb{R}^{m-1}\right) \cong \bigoplus_{j=0}^{\kappa} \mathcal{H}_{h-2 j}\left(\mathbb{R}^{m}\right) \backslash \bigoplus_{j=0}^{\kappa} \mathcal{H}_{h-(2 j+1)}\left(\mathbb{R}^{m}\right) .\end{cases}
$$

Denoting irreducible $\operatorname{Spin}(m)$-representations by their highest weight, it follows that the decomposition of a (homogeneous) HSD kernel space can be computed as follows:

$$
\left\{\begin{array}{l}
h=2 \kappa \quad \mathcal{K}_{h, \lambda} \cong \bigoplus_{j=0}^{\kappa}(h-2 j) \otimes \lambda^{\prime}-\bigoplus_{j=0}^{\kappa-1}(h-2 j-1) \otimes \lambda^{\prime} \\
h=2 \kappa+1 \quad \mathcal{K}_{h, \lambda} \cong \bigoplus_{j=0}^{\kappa}(h-2 j) \otimes \lambda^{\prime}-\bigoplus_{j=0}^{\kappa}(h-2 j-1) \otimes \lambda^{\prime}
\end{array}\right.
$$

Here, the minus sign should be understood on the level of characters of irreducible representations, in which only the weights play a role.After subtraction of the characters in the right hand side of (IV.1), what remains is the character of the left hand side, and this can again be identified with the representation space.

Let us give an example to make this reasoning more clear.

Example IV.2. Take $h=4$. The Fischer decomposition yields

$$
\mathcal{P}_{4}\left(\mathbb{R}^{m-1}, \mathcal{S}_{\lambda}\right) \cong \mathcal{H}_{4}\left(\mathbb{R}^{m-1}, \mathcal{S}_{\lambda}\right) \oplus \mathcal{H}_{2}\left(\mathbb{R}^{m-1}, \mathcal{S}_{\lambda}\right) \oplus \mathcal{H}_{0}\left(\mathbb{R}^{m-1}, \mathcal{S}_{\lambda}\right)
$$


Decomposition of the polynomial kernel of arbitrary higher spin Dirac operators

On the other hand, the branching rules tell us that

$$
\begin{aligned}
\mathcal{H}_{4}\left(\mathbb{R}^{m}, \mathcal{S}_{\lambda}\right) \cong & \mathcal{H}_{0}\left(\mathbb{R}^{m-1}, \mathcal{S}_{\lambda}\right) \oplus \mathcal{H}_{1}\left(\mathbb{R}^{m-1}, \mathcal{S}_{\lambda}\right) \oplus \mathcal{H}_{2}\left(\mathbb{R}^{m-1}, \mathcal{S}_{\lambda}\right) \oplus \mathcal{H}_{3}\left(\mathbb{R}^{m-1}, \mathcal{S}_{\lambda}\right) \\
& \oplus \mathcal{H}_{4}\left(\mathbb{R}^{m-1}, \mathcal{S}_{\lambda}\right) \\
\mathcal{H}_{3}\left(\mathbb{R}^{m}, \mathcal{S}_{\lambda}\right) \cong & \mathcal{H}_{0}\left(\mathbb{R}^{m-1}, \mathcal{S}_{\lambda}\right) \oplus \mathcal{H}_{1}\left(\mathbb{R}^{m-1}, \mathcal{S}_{\lambda}\right) \oplus \mathcal{H}_{2}\left(\mathbb{R}^{m-1}, \mathcal{S}_{\lambda}\right) \oplus \mathcal{H}_{3}\left(\mathbb{R}^{m-1}, \mathcal{S}_{\lambda}\right) \\
\mathcal{H}_{2}\left(\mathbb{R}^{m}, \mathcal{S}_{\lambda}\right) \cong & \mathcal{H}_{0}\left(\mathbb{R}^{m-1}, \mathcal{S}_{\lambda}\right) \oplus \mathcal{H}_{1}\left(\mathbb{R}^{m-1}, \mathcal{S}_{\lambda}\right) \oplus \mathcal{H}_{2}\left(\mathbb{R}^{m-1}, \mathcal{S}_{\lambda}\right) \\
\mathcal{H}_{1}\left(\mathbb{R}^{m}, \mathcal{S}_{\lambda}\right) \cong & \mathcal{H}_{0}\left(\mathbb{R}^{m-1}, \mathcal{S}_{\lambda}\right) \oplus \mathcal{H}_{1}\left(\mathbb{R}^{m-1}, \mathcal{S}_{\lambda}\right) \\
\mathcal{H}_{0}\left(\mathbb{R}^{m}, \mathcal{S}_{\lambda}\right) \cong & \mathcal{H}_{0}\left(\mathbb{R}^{m-1}, \mathcal{S}_{\lambda}\right) .
\end{aligned}
$$

Hence, we indeed find that

$$
\mathcal{K}_{4, \lambda} \cong \bigoplus_{j=0}^{2} \mathcal{H}_{2 j}\left(\mathbb{R}^{m}, \mathcal{S}_{\lambda}\right)-\bigoplus_{j=0}^{1} \mathcal{H}_{2 j+1}\left(\mathbb{R}^{m}, \mathcal{S}_{\lambda}\right)
$$

Let us take a closer look at the tensor products of the form $(k) \otimes \lambda^{\prime}$. According to (III.1), this tensor product decomposes as

$$
(k) \otimes \lambda^{\prime}=\sum_{\zeta}(((k) /(\zeta \cdot Q)) \cdot(\lambda / \zeta))^{\prime}
$$

The Young diagram corresponding to the partition $(k)$ is given by a horizontal line of $k$ boxes:

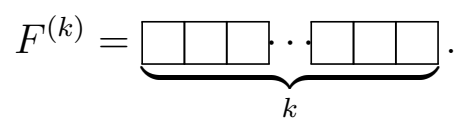

Consequently, the only Young diagrams of the partitions $\eta$ for which $(k) / \eta$ can give a non-trivial result must also be a horizontal line. This means that the only partitions $\zeta$ in $(k) /(\zeta \cdot Q)$ that must be considered have horizontal lines as Young diagrams. Hence in the Littlewood-Richardson product, the only partitions in $\zeta \cdot Q$, where

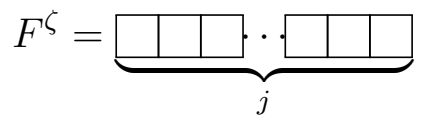

that must be considered are the ones where the Young diagrams are of the form

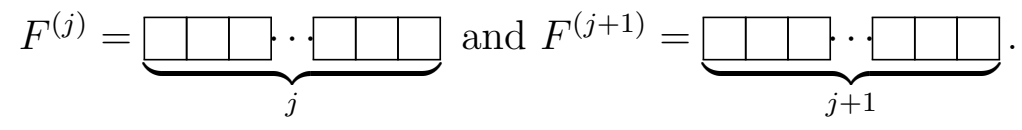


Decomposition of the polynomial kernel of arbitrary higher spin Dirac operators

These are coming from the Littlewood-Richardson products of $\zeta$ with (0), respectively (1). From this it follows that

$$
\begin{aligned}
(k) \otimes \lambda^{\prime} & =\sum_{\zeta}(((k) /((j)+(j+1))) \cdot(\lambda /(j)))^{\prime} \\
& =\sum_{j}(((k) /(j)) \cdot(\lambda /(j)))^{\prime}+\sum_{j}(((k) /(j+1)) \cdot(\lambda /(j)))^{\prime}
\end{aligned}
$$

This result holds for all $k>0 \in \mathbb{N}$. Furthermore, following the division rules, we get in this case that

$$
(k) /(j+1)=(k-1) /(j),
$$

as one always has to remove at least one box from the partition $(k)$ for all $j \in \mathbb{N}$. Using this result, we can simplify (IV.1). Since

$$
\begin{aligned}
(h) \otimes \lambda^{\prime} & =\sum_{j}(((h) /(j)) \cdot(\lambda /(j)))^{\prime}+\sum_{j}(((h-1) /(j)) \cdot(\lambda /(j)))^{\prime} \\
(h-1) \otimes \lambda^{\prime} & =\sum_{j}(((h-1) /(j)) \cdot(\lambda /(j)))^{\prime}+\sum_{j}(((h-2) /(j)) \cdot(\lambda /(j)))^{\prime} \\
(h-2) \otimes \lambda^{\prime} & =\sum_{j}(((h-2) /(j)) \cdot(\lambda /(j)))^{\prime}+\sum_{j}(((h-3) /(j)) \cdot(\lambda /(j)))^{\prime} \\
& \vdots \\
(2) \otimes \lambda^{\prime} & =\sum_{j}(((2) /(j)) \cdot(\lambda /(j)))^{\prime}+\sum_{j}(((1) /(j)) \cdot(\lambda /(j)))^{\prime} \\
(1) \otimes \lambda^{\prime} & =\sum_{j}(((1) /(j)) \cdot(\lambda /(j)))^{\prime}+\sum_{j}(((0) /(j)) \cdot(\lambda /(j)))^{\prime} \\
(0) \otimes \lambda^{\prime} & =\sum_{j}(((0) /(j)) \cdot(\lambda /(j)))^{\prime},
\end{aligned}
$$

and the right-hand sides of equation (IV.1) are obtained by alternately adding and subtracting these expressions, equation (IV.1) reduces to

$$
(h) \otimes \lambda^{\prime}=\sum_{j}(((h) /(j)) \cdot(\lambda /(j)))^{\prime} .
$$

A similar reasoning can be made for the tensor product $(k) \otimes \lambda$. Together with the results in $^{1}$, the decomposition is as follows:

$$
\begin{aligned}
(k) \otimes \lambda & =\sum_{\zeta}((k) / \zeta) \cdot(\lambda / \zeta) \\
& =\sum_{j}((k) /(j)) \cdot(\lambda /(j)) .
\end{aligned}
$$


We notice that this sum is the same as the one appearing in (IV.2), hence we can conclude that

$$
\mathcal{K}_{h, \lambda} \cong((h) \otimes \lambda)^{\prime}
$$

One can simplify the expression as little further using the Littlewood-Richardson division rules:

$$
\begin{aligned}
\mathcal{K}_{h, \lambda} & =\sum_{j=0}^{\infty}(((h) /(j)) \cdot(\lambda /(j)))^{\prime} \\
& =\sum_{j=0}^{\infty}((h-j) \cdot(\lambda /(j)))^{\prime} \\
& =\sum_{j=0}^{\min \left(h, l_{1}\right)}((h-j) \cdot(\lambda /(j)))^{\prime} .
\end{aligned}
$$

We want to know what this sum explicitly decomposes as. Hence, let us calculate the Littlewood-Richardson products explicitly. Since the prime in the sum is a component-wise shift of each highest weight vector appearing in the decomposition, we can omit it without loss of generality, and at the end of the computation add it to each component. So we will investigate the direct sum

$$
\sum_{j=0}^{\min \left(h, l_{1}\right)}(h-j) \cdot(\lambda /(j))
$$

Set $\lambda=\left(l_{1}, \ldots, l_{k}\right)$ and suppose first of all that $h \geq l_{1}$. Since the Young diagram of each $(j)$ is a horizontal line of boxes, The Young diagrams in $\lambda /(j)$ are the ones obtained by removing $j$ boxes from the $F^{\lambda}$, such that in each column only the bottom box can be removed. For instance, if $\lambda=(7,4,2,2,1)$, the only boxes that can be removed are the marked ones:

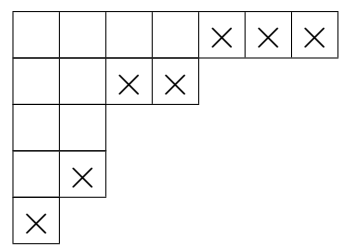

Since the result after removing the boxes must still be a valid Young diagram, if a box is removed on a certain row, all boxes on the same row right of it must be removed as well. This means that in row 1 , at most $l_{1}-l_{2}$ boxes can be removed, in row 2 at most $l_{2}-l_{3}$, $\ldots$, in row $k-1$ at most $l_{k-1}-l_{k}$ and finally in row $k$ at most $l_{k}$. Hence,

$$
\lambda /(j)=\sum_{i_{1}=0}^{l_{1}-l_{2}} \cdots \sum_{i_{k-1}=0}^{l_{k-1}-l_{k}} \sum_{i_{k}=0}^{l_{k}}\left(l_{1}-i_{1}, \ldots, l_{k}-i_{k}\right),
$$


with the condition that $i_{1}+\cdots+i_{k}=j$. This corresponds to the removal of a 'horizontal strip' of length $j^{15}$. Due to (IV.3) and the fact that $h \geq l_{1}$, we must only consider the $j$ 's where $j \leq l_{1}$.

Next, we have to consider the Littlewood-Richardson product with $(h-j)$. Note that since $h \geq l_{1}$, this is always non-trivial. Due to the linearity of this product we have that

$$
(\lambda /(j)) \cdot(h-j)=\sum_{i_{1}=0}^{l_{1}-l_{2}} \ldots \sum_{i_{k-1}=0}^{l_{k-1}-l_{k}} \sum_{i_{k}=0}^{l_{k}}\left(l_{1}-i_{1}, \ldots, l_{k}-i_{k}\right) \cdot(h-j) .
$$

Each partition in $\left(l_{1}-i_{1}, \ldots, l_{k}-i_{k}\right) \cdot(h-j)$ is obtained by adding $h-j$ boxes to the Young diagram of $\left(l_{1}-i_{1}, \ldots, l_{k}-i_{k}\right)$. Following the product rules this means that we can add at most

- $l_{k}-i_{k}$ boxes to row $k+1$;

- $\left(l_{k-1}-i_{k-1}\right)-\left(l_{k}-i_{k}\right)$ boxes to row $k$;

- ...

- $\left(l_{2}-i_{2}\right)-\left(l_{3}-i_{3}\right)$ boxes to row 3 ;

- $\left(l_{1}-i_{1}\right)-\left(l_{2}-i_{2}\right)$ boxes to row 2 ;

- the rest of the $h-j$ boxes to row 1 .

This means that at most $l_{1}-i_{1}$ boxes can be added to rows 2 till $k+1$. Hence at least

$$
(h-j)-\left(l_{1}-i_{1}\right)=h-l_{1}-\sum_{a=2}^{k} i_{a}
$$

boxes must be added to row 1 . The resulting sum can be at most $l_{2}$, so to make sure that this number of boxes is positive, we suppose for now that $h \geq l_{1}+l_{2}$. If this condition is satisfied, we can also make the following interpretation. Fill up rows 2 to $k+1$ of $\left(l_{1}-i_{1}, \ldots, l_{k}-i_{k}\right)$ completely and add the remaining $h-l_{1}-\sum_{a=2}^{k} i_{a}$ boxes to row 1 . This results in the partition

$$
\left(h-\sum_{a=1}^{k} i_{a}, l_{1}-i_{1}, \ldots, l_{k}-i_{k}\right)
$$


Decomposition of the polynomial kernel of arbitrary higher spin Dirac operators

Then all partitions in $\left(l_{1}-i_{1}, \ldots, l_{k}-i_{k}\right) \cdot(h-j)$ are exactly the ones where some of the added boxes in rows 2 to $k+1$ are moved to row 1 . Hence

$$
\begin{aligned}
& \left(l_{1}-i_{1}, \ldots, l_{k}-i_{k}\right) \cdot(h-j) \\
& =\left(l_{1}-i_{1}, \ldots, l_{k}-i_{k}\right) \cdot\left(h-\sum_{a=1}^{k} i_{a}\right) \\
& =\sum_{j_{1}=0}^{\left(l_{1}-i_{1}\right)-\left(l_{2}-i_{2}\right)} \cdots \sum_{j_{k-1}=0}^{\left(l_{k-1}-i_{k-1}\right)-\left(l_{k}-i_{k}\right)} \sum_{j_{k}=0}^{\left(l_{k}-i_{k}\right)}\left(h-\sum_{a=1}^{k} i_{a}+\sum_{a=1}^{k} j_{a}, l_{1}-i_{1}-j_{1}, \ldots, l_{k}-i_{k}-j_{k}\right) .
\end{aligned}
$$

Finally, taking the sum over the $j$ 's in (IV.3) corresponds to removing the condition $i_{1}+$ $\cdots+i_{k}=j$ and taking sums over all possible $i_{1}, \ldots, i_{k}$. This means that, if $h \geq l_{1}+l_{2}$, we get

$$
\mathcal{K}_{h, \lambda}=\sum_{i_{1}=0}^{l_{1}-l_{2}} \cdots \sum_{i_{k}=0}^{l_{k}} \sum_{j_{1}=0}^{\left(l_{1}-i_{1}\right)-\left(l_{2}-i_{2}\right)} \cdots \sum_{j_{k}=0}^{\left(l_{k}-i_{k}\right)}\left(h+\sum_{a=1}^{k}\left(j_{a}-i_{a}\right), l_{1}-i_{1}-j_{1}, \ldots, l_{k}-i_{k}-j_{k}\right)^{\prime} .
$$

Backtracking the proposed conditions on $h$, we also find the full decomposition in the cases where $h<l_{1}+l_{2}$. Let us put our findings in a theorem.

Theorem IV.3. The h-homogeneous polynomial kernel space $\mathcal{K}_{h, \lambda}$ of the higher spin Dirac operator $\mathcal{Q}_{\lambda}$, where $\lambda=\left(l_{1}, \ldots, l_{k}\right)$ decomposes as follows in irreducible modules for Spin $(m)$ :

$$
\mathcal{K}_{h, \lambda} \cong \bigoplus_{i_{1}=0}^{l_{1}-l_{2}} \cdots \bigoplus_{i_{k}=0}^{l_{k}} \bigoplus_{j_{1}=0}^{\left(l_{1}-i_{1}\right)-\left(l_{2}-i_{2}\right)} \cdots \bigoplus_{j_{k}=0}^{\left(l_{k}-i_{k}\right)}\left(h+\sum_{a=1}^{k}\left(j_{a}-i_{a}\right), l_{1}-i_{1}-j_{1}, \ldots, l_{k}-i_{k}-j_{k}\right)^{\prime}
$$

where one has to put two conditions on the summation indices:

$$
\sum_{a=1}^{k} j_{a} \geq l_{1}-h+\sum_{b=2}^{k} i_{b}
$$

and

$$
\sum_{a=1}^{k} i_{a} \leq h
$$

Note that (IV.5) is always satisfied if $h \geq l_{1}+l_{2}$ and (IV.6) is always satisfied if $h \geq l_{1}$.

In the case where $h \geq l_{1}+l_{2}$, this decomposition is exactly the conjecture made in ${ }^{11}$, but were unable to prove without the techniques explained in the current article. Following ${ }^{11}$, we can put some extra structure on this decomposition. The HSD operator (II.1) is in fact a composition of the Dirac operator $\partial_{x}$ and an additional projection operator. This means that we have two types of solutions to $\mathcal{Q}_{\lambda}$ : 
Decomposition of the polynomial kernel of arbitrary higher spin Dirac operators

- Type A solutions: solutions to $\mathcal{Q}_{\lambda}$ which are already in the kernel of $\partial_{x}$.

- Type B solutions: remaining solutions of $\mathcal{Q}_{\lambda}$ that are not of type A.

In the case where $h \geq l_{1}$, it was proven in ${ }^{6}$ that the polynomial space of $h$-homogeneous type A solutions of $\mathcal{Q}_{\lambda}$, denoted by $\mathcal{M}_{h, \lambda}^{s}$, decomposes as

$$
\mathcal{M}_{h, \lambda}^{s} \cong \bigoplus_{j_{1}=0}^{l_{1}-l_{2}} \cdots \bigoplus_{j_{k-1}=0}^{l_{k-1}-l_{k}} \bigoplus_{j_{k}=0}^{l_{k}}\left(h+\sum_{a=1}^{k} j_{i}, l_{1}-j_{1}, \ldots l_{k}-j_{k}\right)^{\prime}
$$

hence

$$
\mathcal{K}_{h, \lambda} \cong \bigoplus_{i_{1}=0}^{l_{1}-l_{2}} \cdots \bigoplus_{i_{k}=0}^{l_{k}} \mathcal{M}_{h-\sum_{a=1}^{s} i_{a}, l_{1}-i_{1}, \ldots, j_{k}-i_{k}}^{s}
$$

which proves the conjecture we made in ${ }^{11}$.

In the case where $\lambda=\left(l_{1}\right)$, we get the full decomposition of the polynomial kernel of the Rarita-Schwinger operator $\mathcal{R}_{l_{1}}$. If $h \geq l_{1}$,

$$
\mathcal{K}_{h, l_{1}} \cong \bigoplus_{i_{1}=0}^{l_{1}} \bigoplus_{j_{1}=0}^{l_{1}-i_{1}} \mathcal{S}_{h+j_{1}-i_{1}, l_{1}-i_{1}-j_{1}}
$$

This is in accordance with the original result obtained $\mathrm{in}^{3}$. We can now extend this result for all $h<l_{1}$ :

$$
\mathcal{K}_{h, l_{1}} \cong \bigoplus_{i_{1}=0}^{h} \bigoplus_{j_{1}=l_{1}-h}^{l_{1}-i_{1}} \mathcal{S}_{h+j_{1}-i_{1}, l_{1}-i_{1}-j_{1}}
$$

due to the conditions (IV.5) and (IV.6).

To conclude this article, let us give some explicit examples of such kernel decompositions.

Example IV.4. First consider the easiest case, where $\lambda=(0)$. Then the higher spin Dirac operator reduces to the classical Dirac operator $\partial_{x}$, whence the direct sum in Theorem IV.3 reduces to $\operatorname{ker}_{h} \partial_{x}=\mathcal{S}_{h}$, the space of $h$-homogeneous monogenic functions, as is well-known.

Example IV.5. If $h=2$ and $\lambda=(4)$, then $\operatorname{ker}_{2} \mathcal{R}_{4}$ consists of the components

$$
\begin{gathered}
(6,0)^{\prime} \\
(5,1)^{\prime}
\end{gathered}
$$


Decomposition of the polynomial kernel of arbitrary higher spin Dirac operators

When we consider a case where $h \geq l_{1}$, say, $h=5$ and $\lambda=(3)$, the kernel $\operatorname{ker}_{5} \mathcal{R}_{3}$ decomposes as
$(8,0)^{\prime}$
$(6,0)^{\prime}$
$(4,0)^{\prime}$
$(2,0)^{\prime}$
$(7,1)^{\prime}$
$(5,1)^{\prime}$
$(3,1)^{\prime}$
$(6,2)^{\prime}$
$(4,2)^{\prime}$
$(5,3)^{\prime}$

We recognize the triangular structure mentioned in ${ }^{3}$.

Example IV.6. When we take the length $k$ of $\lambda$ equal to 2 , we get for $h=2$ and $\lambda=(4,1)$, we get the decomposition

$$
\begin{array}{ccc}
(5,0,0)^{\prime} & (3,0,0)^{\prime} \\
(5,1,0)^{\prime} & 2(4,1,0)^{\prime} & (2,1,0)^{\prime} \\
& & (3,2,0)^{\prime} \\
(5,1,1)^{\prime} & (3,1,1)^{\prime} \\
& (4,2,1)^{\prime}
\end{array}
$$

A case where $h \geq l_{1}+l_{2}$, for instance taking $h=4$ and $\lambda=(2,1)$ results in a decomposition

$$
\begin{aligned}
& (6,0,0)^{\prime} \quad(4,0,0)^{\prime} \quad(2,0,0)^{\prime} \\
& (7,1,0)^{\prime} \quad 2(5,1,0)^{\prime} \quad 2(3,1,0)^{\prime} \quad(1,1,0)^{\prime} \\
& (6,2,0)^{\prime} \quad 2(4,2,0)^{\prime} \quad(2,2,0)^{\prime} \\
& (5,3,0)^{\prime} \quad(3,3,0)^{\prime} \\
& (6,1,1)^{\prime} \quad(4,1,1)^{\prime} \quad(2,1,1)^{\prime} \\
& (5,2,1)^{\prime} \quad(3,2,1)^{\prime} \\
& (4,3,1)^{\prime}
\end{aligned}
$$

\section{CONCLUSION}

In this article we managed to fully decompose the polynomial kernel space of a general HSD operator into irreducible modules for the spin group. We hereby found a closed formula for the $h$-homogeneous kernel space. 
Decomposition of the polynomial kernel of arbitrary higher spin Dirac operators

\section{REFERENCES}

${ }^{1}$ Black, G.R.E, King, R.C, Wybourne, B.G., Kronecker products for compact semisimple Lie groups, J. Phys. A: Math. Gen. 16, pp. 1555-1589 (1983).

${ }^{2}$ Brackx, F., Delanghe, R., Sommen, F., Clifford Analysis, Research Notes in Mathematics 76, Pitman, London (1982).

${ }^{3}$ Bureš, J., Sommen, F., Souček, V., Van Lancker, P., Rarita-Schwinger type operators in Clifford analysis, Journal of Funct. Anal. 185, pp. 425-456 (2001).

${ }^{4}$ Bureš, J., Sommen, F., Souček, V., Van Lancker, P., Symmetric analogues of RaritaSchwinger equations, Ann. Glob. Anal. Geom. 21 No. 3, pp. 215-240 (2001).

${ }^{5}$ Constales, D., Sommen. F., Van Lancker, P., Models for irreducible representations of $\operatorname{Spin}(m)$, Adv. Appl. Clifford Algebras 11 No. S1, pp. 271-289 (2001).

${ }^{6}$ De Schepper, H., Eelbode, D., Raeymaekers, T., On a special type of solutions for arbitrary higher spin Dirac Operators, J. Phys. A: Math. Theor. 43 No. 32, pp. 1-13 (2010).

${ }^{7}$ De Schepper, H., Eelbode, D., Raeymaekers, T., Twisted higher spin Dirac operators, Complex Anal. Oper. Theory 8, pp. 429-447 (2014).

${ }^{8}$ Delanghe, R., Sommen, F., Souček, V., Clifford analysis and spinor valued functions, Kluwer Academic Publishers, Dordrecht (1992).

${ }^{9}$ Dirac, P.A.M, The quantum theory of the electron, Proceedings of the Royal Society of London 117, (1928).

${ }^{10}$ Eelbode, D., Raeymaekers, T., Construction of higher spin operators using transvector algebras, J. Math. Phys. 55, 101703 (2014).

${ }^{11}$ Eelbode, D., Raeymaekers, T., Polynomial solutions for arbitrary higher spin Dirac operators, Exp. Math.

${ }^{12}$ Gilbert, J., Murray, M.A.M., Clifford algebras and Dirac operators in harmonic analysis, Cambridge University Press, Cambridge (1991).

${ }^{13}$ Lascoux, A., Pragacz, P., S-function series, J. Phys. A: Math. Gen. 21, pp. 4105-4114 (1988).

${ }^{14}$ Littlewood, D.E., Richardson, A.R., Group Characters and Algebra, Phil. Trans. R. Soc. A 133, pp. 99-141 (1934).

${ }^{15}$ Macdonald, I.G., Symmetric functions and Hall Polynomials, Oxford Math. Monographs, Clarendon Press Oxford (1979). 
Decomposition of the polynomial kernel of arbitrary higher spin Dirac operators

${ }^{16}$ Rarita, W., Schwinger, J., On a theory of particles with half-integer spin, Phys. Rev. 60, p. 61 (1941). 